Journal of

Molecular Microbiology

and Biotechnology
J Mol Microbiol Biotechnol 2010;19:117-122

DOI: $\underline{10.1159 / 000321497}$

\title{
High Efficiency of a Sequential Recombinase-Mediated Cassette Exchange Reaction in Escherichia coli
}

\author{
Natalia Malchin ${ }^{a}$ Tatiana Molotsky ${ }^{a}$ Ilya Borovok ${ }^{b}$ Yuri Voziyanov ${ }^{c}$ \\ Alexander B. Kotlyar ${ }^{a}$ Ezra Yagil ${ }^{\mathrm{a}}$ Mikhail Kolot $^{\mathrm{a}}$ \\ Departments of a Biochemistry and ${ }^{\mathrm{b}}$ Molecular Microbiology and Biotechnology, Tel Aviv University, Tel Aviv, Israel; \\ 'School of Biological Sciences/IfM, Louisiana Tech University, Ruston, La., USA
}

\section{Key Words}

Site-specific recombination - Flp recombinase $\cdot$ Coliphage HK022 - Integrase $\cdot$ Recombinase-mediated cassette exchange $\cdot$ Atomic force microscopy

\begin{abstract}
A comparison between the efficiency of recombinase-mediated cassette exchange (RMCE) reactions catalyzed in Escherichia coli by the site-specific recombinases Flp of yeast and Int of coliphage $\mathrm{HKO} 22$ has revealed that an Flp-catalyzed $\mathrm{RMCE}$ reaction is more efficient than an Int-HK022 catalyzed reaction. In contrast, an RMCE reaction with 1 pair of frt sites and 1 pair of att sites catalyzed in the presence of both recombinases is very inefficient. However, the same reaction catalyzed by each recombinase individually supplied in a sequential order is very efficient, regardless of the order. Atomic force microscopy images of Flp with its DNA substrates show that only 1 pair of recombination sites forms a synaptic complex with the recombinase. The results suggest that the $\mathrm{RMCE}$ reaction is sequential.

Copyright ๑ 2010 S. Karger AG, Basel
\end{abstract}

\section{Introduction}

Recombinase-mediated cassette exchange (RMCE) technology has become an important biotechnological tool for gene exchange in the eukarya, avoiding the insertion of unnecessary vector DNA or undesired antibioticresistance markers. With this technology 1 or 2 relevant site-specific recombinases catalyze the exchange of an introduced DNA fragment located on an incoming plasmid with a genomic DNA fragment, both flanked by 2 relevant site-specific recombination sites. The most abundant site-specific recombinases used in RMCE reactions are Cre of coliphage P1, Flp of yeast, and Integrase (Int) of the Streptomyces phage $\Phi C 31$. Since RMCE reactions are obtained with a rather low frequency, they usually require some selection force which may be a suicide agent like ganciclovir, counter-selection or promoter trapping. In case only 1 site-specific recombinase is employed in an RMCE reaction, the 2 pairs of flanking recombination sites whose required incompatibility is not always complete must be heterologous [Branda and Dymecki, 2004; Qiao et al., 2009; Sorrell and Kolb, 2005; Wirth et al., 2007].

Despite their requirements of accessory proteins [integration host factor (IHF) and excisionase (Xis)] the site-

Tel. +972 3640 9823, Fax +972 3640 6834, E-Mail kolott@ post.tau.ac.il 


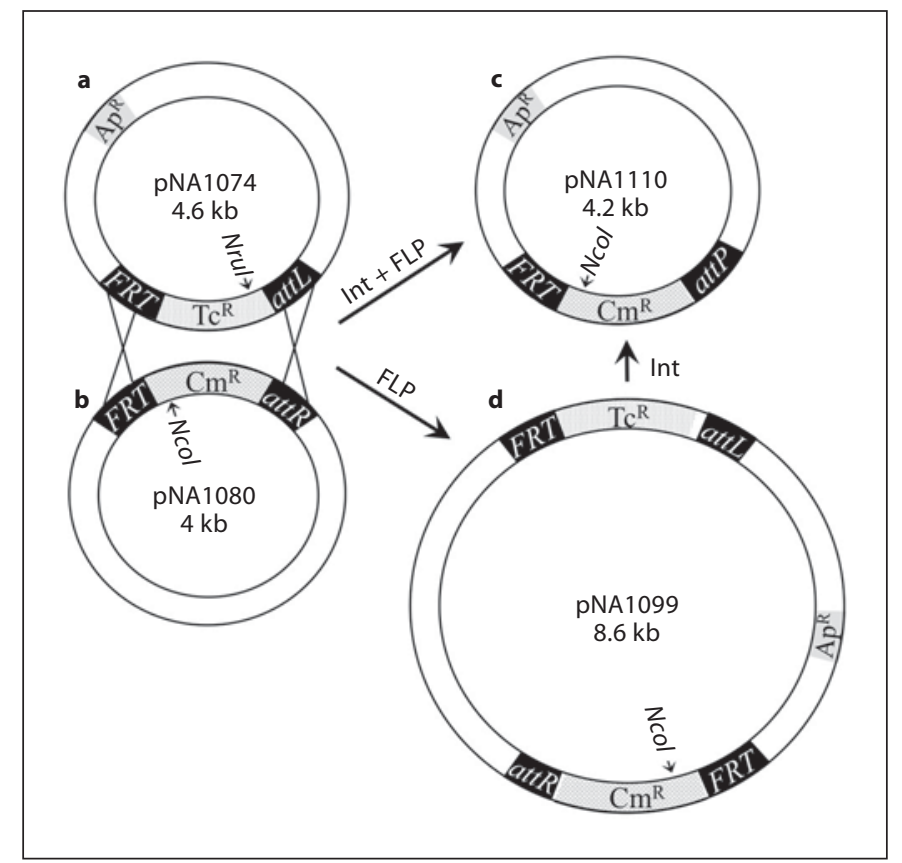

Fig. 1. Schematic representation of the RMCE reaction. The 2 substrates (a and $\mathbf{b}$ ) where 1 pair of recombining sites is frt and the other is attL and attR, as well as the RMCE product (c) and the dimeric intermediate $(\mathbf{d})$ are presented. Only selectable $A p^{R} C^{R}$ products are shown.

specific Int recombinases of coliphage $\lambda$ [Azaro and Landy, 2002] and its relative, coliphage HK022 [Weisberg et al., 1999], have been shown to be active in site-specific integrative $(a t t B \times a t t P)$ as well as excisive $(a t t L \times a t t R)$ reactions in eukaryotic cell cultures and in plants, without the need to supply any accessory protein [Christ and Dröge, 2002; Gottfried et al., 2005; Harel-Levi et al., 2008]. Nevertheless, the presence of IHF and/or Xis in human cells improves these reactions [Corona et al., 2003; Malchin et al., 2009]. In the commercially developed Gateway system used for gene cloning (Invitrogen), a modified Int $-\lambda$ replaces restriction enzymes in in vitro RMCE-type reactions. In an attempt to test the ability of Int-HK022 to catalyze an RMCE reaction between 2 plasmids in Escherichia coli in vivo, we have shown that such reactions are detectable, albeit with a rather low frequency [Malchin et al., 2008].

The low frequency of RMCE reactions might be due to one recombinase-DNA synaptic complex interfering with the formation or with the activity of the second synaptic complex. In the former case only 1 synaptic complex between the recombinase and the 2 recombining sites is formed at a time; recombination leads to the formation of an intermediate that carries the integrated plasmid and 2 pairs of sites in the cis configuration (all sites on the same molecule). A subsequent recombination between the second pair of sites leads to the RMCE product. However, a second recombination between the first pair reverts the reaction to the original substrates. If the latter event is more favorable, it may be the reason for the low frequency of the RMCE products. The sequential application of 2 different recombinases in an RMCE reaction may overcome this obstacle, and in the present study we show that this is indeed the case. Such results may have implications in the improvement of RMCE reactions.

\section{Results and Discussion}

\section{Setup of the RMCE Reactions}

In vivo RMCE reactions in E. coli were performed as previously described [Malchin et al., 2008]. An E. coli recF strain (DS941) that carries a kanamycin-resistant $\left(\mathrm{Km}^{\mathrm{R}}\right)$ plasmid expressing the relevant recombinase(s) was cotransformed with 2 plasmids comprising the RMCE substrates that are compatible with the $\mathrm{Km}^{\mathrm{R}}$ recombinase plasmid. Figure 1a, b represents the substrates of one of the reactions in which 1 pair of recombining sites comprised $2 \mathrm{frt}$ sites of Flp and the other pair consisted of 2 compatible att sites of Int. In one of the substrates (pNA1074; fig. 1a), the tetracycline-resistant $\left(\mathrm{Tc}^{\mathrm{R}}\right)$ gene is flanked by 2 recombination sites, i.e. frt and $a t t L$, and it also carries an ampicillin-resistant $\left(\mathrm{Ap}^{\mathrm{R}}\right)$ gene. In the other substrate (pNA1080; fig. 1b) the chloramphenicolresistant $\left(\mathrm{Cm}^{\mathrm{R}}\right)$ gene is flanked by frt and attR that are compatible with the aforementioned sites. As one result of an exchange between the $\mathrm{Tc}^{\mathrm{R}}$ and $\mathrm{Cm}^{\mathrm{R}}$ markers 1 product of the RMCE reaction that is selectable is expected to be of the $\mathrm{Ap}^{\mathrm{R}} \mathrm{Cm}^{\mathrm{R}} \mathrm{Tc}^{\mathrm{S}}$ phenotype (fig. 1c). The recF cells transformed with all 3 plasmids were plated on $\mathrm{Km}+\mathrm{Ap}$ $+\mathrm{Cm}$, and plasmid extract from a pool of many (over 200) $\mathrm{Km}^{\mathrm{R}} \mathrm{Ap}^{\mathrm{R}} \mathrm{Cm}^{\mathrm{R}}$ transformants was used to retransform the original untransformed recF strain and plated on Ap. Single $\mathrm{Ap}^{\mathrm{R}}$ colonies were picked onto individual Ap, Cm, and Tc plates to determine their phenotype. All experiments were performed twice; they also included negative controls in the absence of a recombinase that did not show any RMCE product.

\section{Flp-Catalyzed RMCE Reaction}

We first tested the ability of Flp to catalyze the RMCE reaction in E. coli using its more active Flpe derivative [Buchholz et al., 2008]. The recF strain transformed with 
Table 1. Frequency of RMCE products

\begin{tabular}{|c|c|c|c|c|}
\hline & Recombinase & Sites on substrate & RMCE frequency & RMCE, \% \\
\hline \multirow[t]{2}{*}{ A } & Int & $\begin{array}{l}a t t B-a t t B \times a t t P-a t t P \\
\text { or }\end{array}$ & [Malchin et al., 2008] & 3.5 \\
\hline & & $a t t L-a t t L \times a t t R-a t t R$ & [Malchin et al., 2008] & 5 \\
\hline B & Flp + Int & $f r t-f r t \times f r t-f r t$ & $25 / 100,30 / 100$ & 27.5 \\
\hline $\mathrm{C}$ & Flp + Int & $f r t-a t t L \times f r t-a t t R$ & $1 / 400,1 / 370$ & 0.26 \\
\hline $\mathrm{D}$ & Int & frt-attL - frt-attR (dimer) & $100 / 100,100 / 100$ & 100 \\
\hline E & Flp $\rightarrow$ Int & $f r t-a t t L \times f r t-a t t R$ & $100 / 100,100 / 100$ & 100 \\
\hline $\mathrm{F}$ & Int $\rightarrow$ Flp & $f r t-a t t L \times f r t-a t t R$ & $100 / 100,95 / 100$ & 97.5 \\
\hline
\end{tabular}

Arrows indicate the order of the supplied recombinases.

a plasmid (pNA1086) that expresses both Flp and Int was cotransformed with 2 RMCE substrate plasmids, i.e. one (pNA1079) that was $\mathrm{Ap}^{\mathrm{R}} \mathrm{Tc}^{\mathrm{R}}$ whose $\mathrm{Tc}^{\mathrm{R}}$ gene was flanked by 2 inverted 34-bp minimal frt sites and another (pNA1081) whose $\mathrm{Cm}^{\mathrm{R}}$ gene was flanked by the same $\mathrm{frt}$ sites. Following the procedure previously described, of 2 $\times 100 \mathrm{Ap}^{\mathrm{R}}$ colonies picked from 2 independent experiments, 25 and 30 colonies, respectively, showed the expected RMCE $\mathrm{Ap}^{\mathrm{R}} \mathrm{Cm}^{\mathrm{R}} \mathrm{Tc}^{\mathrm{S}}$ phenotype (table 1, line B). Restriction analysis of 13 such colonies confirmed the exchange between the $\mathrm{Tc}^{\mathrm{R}}$ and $\mathrm{Cm}^{\mathrm{R}}$ genes, and the presence of the frt sites that flank the $\mathrm{Cm}^{\mathrm{R}}$ gene was confirmed by sequencing of 3 such colonies (not shown). The phenotype of the remaining $A p^{R}$ transformants showed that most of them (74 and 68) were $\mathrm{Ap}^{\mathrm{R}} \mathrm{Cm}^{\mathrm{S}} \mathrm{Tc}^{\mathrm{R}}$ (i.e. carried the parental plasmid pNA1079) and only a few (1 and 2) were $\mathrm{Ap}^{\mathrm{R}} \mathrm{Cm}^{\mathrm{R}} \mathrm{Tc}^{\mathrm{R}}$ (i.e. carried both substrates). The $27.5 \%$ average efficiency of Flp (table 1, line B) in the present RMCE reaction proved to be $\sim 5$ - to 10 -fold more efficient than Int in the analogous RMCE reactions (table 1 , line A) that in a previous study showed only 3.5-5\% RMCE products [Malchin et al., 2008].

\section{RMCE Catalysis by the 2 Recombinases}

To test the efficiency of the RMCE reaction catalyzed simultaneously by both Flp and Int, the substrate plasmids shown in figure 1a, b were used; one recombining site was a pair of $f r t$ sites and the second was an attL and attR pair. The recF strain that expressed both recombinases was cotransformed with both substrate plasmids followed by the RMCE detection procedure previously described. Of the $2 \times 100 \mathrm{Ap}^{\mathrm{R}}$ colonies tested none showed an RMCE product, which lead us to extend the analysis to $400 \mathrm{Ap}^{\mathrm{R}}$ colonies in one of the experiments and $370 \mathrm{Ap}^{\mathrm{R}}$ in the repetitive one. Among the $770 \mathrm{Ap}^{\mathrm{R}}$ colonies tested only $2(1+1)$ showed the RMCE $\mathrm{Ap}^{\mathrm{R}} \mathrm{Cm}^{\mathrm{R}}$

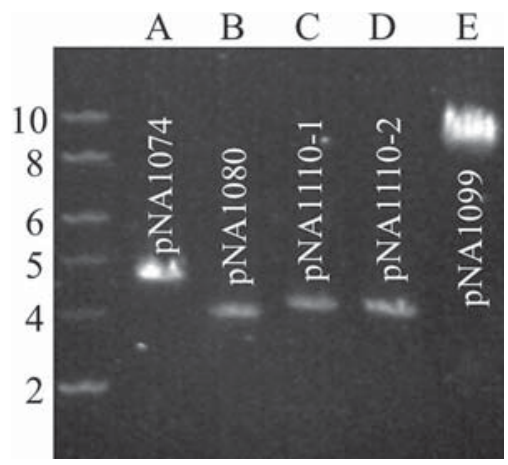

Fig. 2. Gel electrophoresis of the RMCE reaction substrates and products. The $2 \mathrm{RMCE}$ substrates (A and $\mathrm{B}$ ) shown in figure 1 cut with NruI and NcoI, respectively. The 2 RMCE products (C and D) cut with NcoI. E The dimer cut with NcoI.

$\mathrm{Tc}^{\mathrm{S}}$ phenotype (fig. 1c; table 1, line C). Plasmid analyses of these 2 strains confirmed the RMCE product by restriction (fig. 2, lanes $\mathrm{C}$ and $\mathrm{D}$ ) as well as by the sequence (not shown) that confirmed the frt and attP sites flanking the $\mathrm{Cm}^{\mathrm{R}}$ gene (fig. 1c). All the rest of the $\mathrm{Ap}^{\mathrm{R}}$ colonies, except for 1 , carried the $A p^{R} \mathrm{Cm}^{\mathrm{S}} \mathrm{Tc}^{\mathrm{R}}$ phenotype of the $A p^{R}$ parent pNA1074 (fig. 1a). Plasmid extracted from the single colony that was resistant to all 3 antibiotics $\left(A p^{R}\right.$ $\mathrm{Cm}^{\mathrm{R}} \mathrm{Tc}^{\mathrm{R}}$ ) proved to be a dimer of the 2 substrates that resulted from a recombination event between the $2 \mathrm{frt}$ sites (plasmid pNA1099, fig. 1d) as confirmed by restriction (fig. 2, lane E) and by the sequence (not shown) that revealed its 4 recombination sites (fig. 1d). The considerably low efficiency of RMCE catalysis in the presence of both recombinases lead us to suspect that their coexistence may hinder the RMCE reaction in such a way that the protein-DNA complex of one of them interferes with ability of the other to form the second protein-DNA complex. Figure 3 is an RT-PCR analysis of recF cells that ex- 
Fig. 3. RT-PCR products showing the expression of Int, Flp (each alone), and both recombinases together. Each reaction includes the 16S RNA as the internal control. The last 3 columns $(\mathrm{a}-\mathrm{c})$ show the PCR products of the $16 \mathrm{~S}$, int, and Flp using DNA of $E$. coli carrying pNA1086 as a template. $\mathrm{RT}=$ Reverse transcriptase.

\begin{tabular}{|c|c|c|c|c|c|c|c|c|c|c|c|c|c|c|c|}
\hline \multirow{3}{*}{$\begin{array}{c}\text { Plasmid } \\
\text { Primers } \\
\text { RT }\end{array}$} & \multicolumn{3}{|c|}{ pMK155 (Int) } & \multicolumn{3}{|c|}{ pNA1100 (FLP) } & \multirow{3}{*}{$\begin{array}{l}M \\
W\end{array}$} & \multicolumn{4}{|c|}{ pNA1086 (Int + FLP) } & \multirow{3}{*}{$\begin{array}{l}M \\
W\end{array}$} & \multicolumn{3}{|c|}{$\begin{array}{c}\text { E. coli } \\
\text { (pNA1086) }\end{array}$} \\
\hline & $16 \mathrm{~S}$ & \multicolumn{2}{|c|}{ Int } & $16 \mathrm{~S}$ & \multicolumn{2}{|c|}{ FLP } & & $16 \mathrm{~S}$ & Int & \multicolumn{2}{|c|}{ FLP } & & a & $b$ & c \\
\hline & $-\quad+$ & - & + & $-1+$ & - & + & & $-1+$ & $-1+$ & - & + & & & PCR & \\
\hline $\begin{array}{l}0.5 \rightarrow \\
0.4 \\
0.3 \rightarrow \\
0.2 \rightarrow\end{array}$ & $=$ & & $=$ & - & & - & $\underline{0}$ & $=$ & - & & 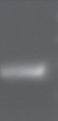 & - & & - & - \\
\hline
\end{tabular}

press each recombinase separately or both of them together, using the 16S rRNA genes as an internal control. It shows that in all cases both recombinases are similarly expressed.

The dimer shown in figure $1 \mathrm{~d}$ was presumably formed owing to the more efficient Flp recombinase activity leaving the $a t t L$ and $a t t R$ sites of Int unrecombined. To test the efficiency of Int in using this dimer as a substrate to form the RMCE product, $r e c F$ cells that carried the Intexpressing $\mathrm{Km}^{\mathrm{R}}$ plasmid $\mathrm{pMK} 155$ were transformed with this dimer and followed through the RMCE detection procedure. Of the $2 \times 100$ picked $\mathrm{Ap}^{\mathrm{R}}$ transformants all 200 showed the expected RMCE $\mathrm{Ap}^{\mathrm{R}} \mathrm{Cm}^{\mathrm{R}} \mathrm{Tc}^{\mathrm{S}}$ phenotype (table 1, line D); this was confirmed by restriction and sequence in one of them (not shown). This result supports our previous suggestion that the formation of an RMCE product may result from a sequential reaction rather than a simultaneous one, i.e. by forming a dimer followed by its resolution [Malchin et al., 2008]. These results suggest, again, that the simultaneous presence of the 2 recombinases somehow interferes with the RMCE reaction (at least in bacteria). It should also be mentioned that in all of the Int-catalyzed at $L \times$ att $R$ reactions reported here no Xis was supplied because we noticed that the presence of Xis, as previously reported [Malchin et al., 2008], did not improve the reaction (not shown). Apparently the Fis protein, supplied by the host, was sufficient [Thompson et al., 1987].

\section{High Efficiency of Sequential RMCE Reactions}

To test our suggestion that a sequential RMCE reaction might be more efficient than a simultaneous one, we repeated the RMCE reaction using the same plasmids as substrates (fig. 1a, b), following a protocol that supplies each recombinase in a sequential order. $R e c F$ cells expressing a single recombinase were cotransformed with the substrates, and plasmid extract from a pool of $\mathrm{Km}^{\mathrm{R}}$
$\mathrm{Ap}^{\mathrm{R}} \mathrm{Cm}^{\mathrm{R}}$ transformants was used to transform the $\mathrm{recF}$ host and plate for $\mathrm{Ap}^{\mathrm{R}} \mathrm{Cm}^{\mathrm{R}}$ colonies in order to get rid of the first recombinase located on the $\mathrm{Km}^{\mathrm{R}}$ vector. Next, plasmid prepared from a pool of the $\mathrm{Ap}^{\mathrm{R}} \mathrm{Cm}^{\mathrm{R}}$ colonies was used to transform the recF host that expressed the second recombinase. Plasmid extract from pooled $\mathrm{Km}^{\mathrm{R}}$ $\mathrm{Ap}^{\mathrm{R}} \mathrm{Cm}^{\mathrm{R}}$ colonies was then used to transform the recF host and plate for $\mathrm{Ap}^{\mathrm{R}}$ colonies. When Flp was supplied as the first recombinase (plasmid pNA1110) and Int as the second (plasmid pMK155), all $2 \times 100 \mathrm{Ap}^{\mathrm{R}}$ colonies picked carried the $\mathrm{Ap}^{\mathrm{R}} \mathrm{Cm}^{\mathrm{R}} \mathrm{Tc}^{\mathrm{S}}$ RMCE phenotype (table 1 , line $\mathrm{E}$ ). In the reverse order, i.e. when Int was supplied first and Flp second, of the $2 \times 100 \mathrm{Ap}^{\mathrm{R}}$ colonies 100 and 95 showed the expected RMCE phenotype (table 1 , line F). In each of the 2 experiments the RMCE phenotype of 7 recombinant plasmids was confirmed by restriction and in 2 of them by sequence (not shown). The results have clearly demonstrated that in a sequential treatment the RMCE reaction is by far more efficient than a simultaneous one.

\section{Visualization of Synaptic Complexes}

In our previous atomic force microscopy (AFM) study with Int alone [Malchin et al., 2008] we could observe $\sim 5 \%$ DNA-protein complexes in all of which the 2 substrates formed only 1 synaptic complex with Int via a single pair of att sites. We could not find any image of a complete RMCE complex where both pairs of att sites were occupied by Int. This led us to assume that the $\mathrm{RMCE}$ reaction is sequential, i.e. that only 1 Int-att complex is formed at a time. However, with the low efficiency of the Int-catalyzed RMCE reaction (3.5-5\%; table 1, line A) the lack of complete RMCE complexes could have been incidental. Since with Flp alone the frequency was over 5 - to 10 -fold higher (table 1 , line $\mathrm{B}$ ), we have repeated the AFM study of an in vitro reaction using an inactive Flp mutant (FlpY343F) whose active Tyr343 was re- 

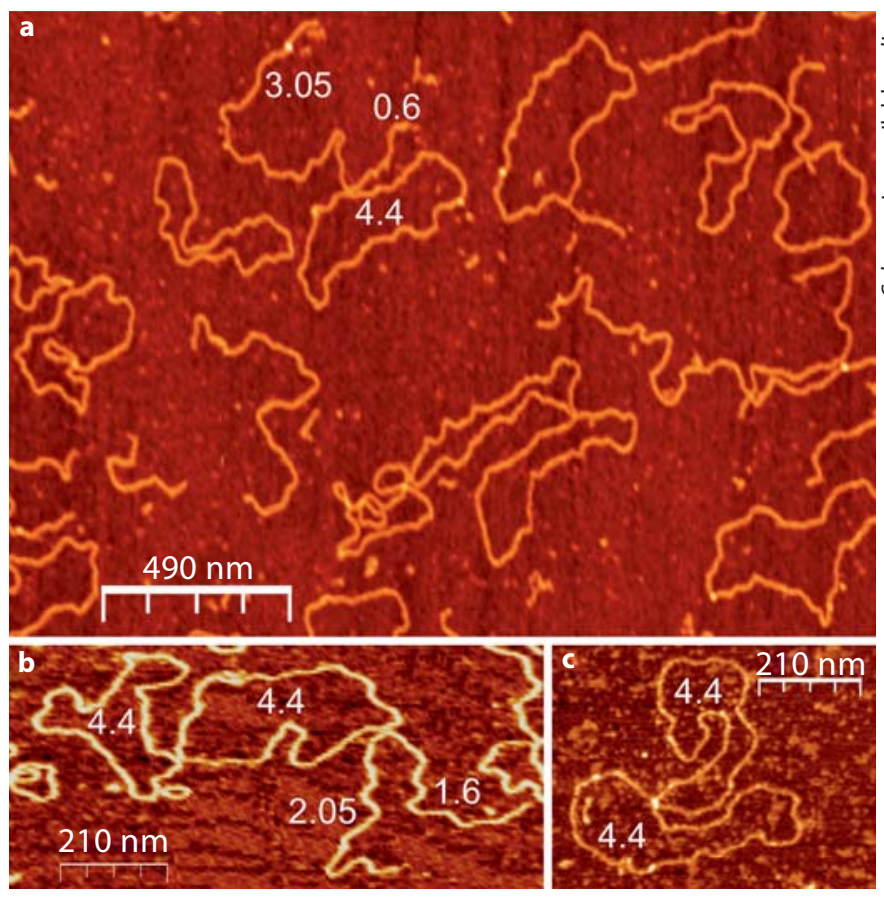

Fig. 4. a AFM image of the in vitro RMCE reaction between the DraIII-linearized plasmid pNA1081 and circular plasmid pNA1079 showing DNA substrates, proteins (bright spots), and a synaptic complex between the circular and linear substrates. b A selected complex between the circular and linear substrates and between 2 circular plasmids. c A selected complex between 2 circular substrates. Numbers indicate approximate sizes in $\mathrm{kb}$.

placed with a Phe residue. The substrates in the reaction (pNA1079 and pNA1081) carried 2 inverted pairs of $f r t$ sites, the latter linearized with DraIII (fig. 4a). About $10 \%$ of the circles were complexed either with the linear substrates (fig. $4 \mathrm{a}, \mathrm{b}$ ) or between 2 circles (fig. 4b, c). Also here, among hundreds of DNA molecules scored, we could not identify a single complete RMCE image, suggesting again that they are not formed. This strengthens our assumption that only 1 synaptic complex is formed at a time. The location of the DraIII site allowed us to distinguish between the 2 possible types of circular-linear synaptic complexes of Flp with a single pair of $f r t$ sites, i.e. one that yielded 2 arms 3.05 and $0.6 \mathrm{~kb}$ long (fig. 4a) and another with 2 arms 2.05 and $1.6 \mathrm{~kb}$ long (fig. 4b). Among the 25 complete complexes scored, we found 7 DNA-Flp complexes of the first type, 6 complexes of the second type, and 12 complexes between 2 circles (fig. 4b, c), one of which was bound in its second frt pair to the linear substrate (fig. $4 \mathrm{~b}$ ). These results indicated that, at least on the linear substrate, there is no preference for a particular frt site to synapse. When the FlpF mutant interacted with the substrates carrying 1 pair of frt sites and the second pair of att sites (fig. 1a, b), we saw similar complexes (not shown). However, when we added the IntF (Y342F) mutant [Malchin et al., 2008] and its 2 accessory proteins IHF and Xis to the mixture, no single complex was found. Instead, we saw larger aggregates of proteins (not shown) that may be complexes between the proteins themselves.

\section{Conclusions}

We have shown that in E. coli an in vivo Flp-catalyzed RMCE reaction is more efficient than an identical Intcatalyzed reaction. When using the 2 recombinases simultaneously the reaction is even less efficient than with Int alone. On the contrary, in a sequential treatment the reaction becomes far more efficient, regardless of the order of the supplied recombinases. The reason is probably that with the use of 2 different recombinases in a sequential manner the option of the undesired reverse reaction that leads to the original substrates is diminished. A sequential protocol using 2 different recombinases in eukaryotic RMCE trials may also improve the reaction except for cell lines which cannot tolerate more than 1 round of transfection. Because in this AFM study and in the one with Int [Malchin et al., 2008] only single synaptic complexes were observed and complete RMCE images that showed 2 synapses within a single pair of substrates could not be found, we assume that any RMCE reaction is sequential, first between 1 pair of sites forming an intermediate, followed by recombination within the second pair that resolves the intermediate into the final RMCE product. The sequential reaction may have an advantage because in a direct RMCE reaction both recombination reactions are in the trans configuration (each member of a pair of recombining sites is on a different DNA molecule), whereas in a sequential reaction the resolution of the intermediate (the dimer in this study) is in cis, a configuration that proved to be more efficient than a trans reaction in E. coli [Malchin et al., 2008] as well as in human cells [Harel-Levi et al. 2008; Kolot et al., 2003; Malchin et al., 2009]. The reduced activity in the RMCE reaction in the presence of both Int and Flp may be owing to the small interval (1-1.4 kb) between the 2 pairs of recombination sites, whereas larger intervals may overcome it; Cre-mediated RMCE reactions between very large intervals (over $100 \mathrm{~kb}$ ) in mouse cells proved successful [Wallace et al., 2007]. Alternatively, by forming protein aggre- 
gates, this reduced activity may be specific to the combination of Flp and Int; when Flp and Cre were used together in an RMCE reaction in mammalian cells, it proved to be rather efficient [Lauth et al., 2007]. Experiments that test RMCE reactions catalyzed by Flp and IntHK022 recombinases in mammalian cells are in progress.

\section{Experimental Procedures}

Bacteria, Growth Conditions, Plasmids, and Oligomers

E. coli K12 recF (strain DS941) [Summers and Sherratt, 1988] served as the bacterial host. Strain NA889 is the recF strain transformed with pMK155. Plasmid transformations were performed by electroporation [Sambrook et al., 1989]. The plasmids and oligomers used are listed in online supplementary table 1 (www. karger.com/doi/10.1159/000321497).

\section{Construction of the FlpY343F Mutant Recombinase}

The FlpY343F mutant recombinase was constructed with a QuickChange Site-Directed Mutagenesis Kit (Stratagene) using the 2 oligomers FY343F-F and FY343F-R.
RT-PCR Analysis

RNA was purified from $3 \mathrm{ml}$ logarithmically growing bacteria at a density of $\mathrm{A}_{600 \mathrm{~nm}}=0.7$ using a Promega SV total RNA isolation kit. RT reactions in the presence and absence of reverse transcriptase were performed with a Verso ${ }^{\mathrm{TM}} \mathrm{CDNA}$ kit (Thermo Scientific) using $1 \mu \mathrm{g}$ RNA in each reaction.

Purification of the FlpY343F Mutant Recombinase

The FlpY343F mutant recombinase was induced overnight with IPTG at $16^{\circ} \mathrm{C}$ and purified using a Ni column (Novagen).

\section{AFM Imaging}

The construction of the protein-DNA complexes, the adsorption of the reaction products onto mica surfaces, and the AFM imaging were performed as previously described [Malchin et al., 2008].

\section{Acknowledgments}

We thank Prof. Phoebe Rice for sending plasmid pET24d. N.M. is supported by a Leon Reich scholarship fund. This research was supported by research grant No. MB-8713-08 from BARD, the United States-Israel Binational Agricultural Research and Development Fund, to E.Y. and M.K and by grant No. RO1GM085848 from the US National Institutes of Health to Y.V.

\section{References}

Azaro MA, Landy A: Integrase and the $\lambda$ int family; in Craig NL, Craigie R, Gellert M, Lambowitz A (eds): Mobile DNAII. Washington, ASM, 2002, pp 118-148

Branda CS, Dymecki SM: Talking about a revolution: The impact of site-specific recombinases on genetic analyses in mice. Dev Cell 2004;6:7-28.

Buchholz F, Angrand PO, Stewart AF: Improved properties of FLP recombinase evolved by cycling mutagenesis. Nat Biotechnol 1998; 16:657-662.

Chen Y, Rice PA: The role of the conserved Trp330 in Flp-mediated recombination: functional and structural analysis. J Biol Chem 2003;278:24800-24807.

-Christ N, Dröge P: Genetic manipulation of mouse embryonic stem cells by mutant lambda integrase. Genesis 2002;32:203-208.

-Corona T, Bao Q, Christ N, Schwartz T, Li J, Dröge P: Activation of site-specific DNA integration in human cells by a single chain integration host factor. Nucleic Acids Res. 2003;31:5140-5148.

-Gottfried P, Lotan O, Kolot M, Maslenin L, Bendov R, Gorovits R, Yesodi V, Yagil E, Rosner A: Site-specific recombination in Arabidopsis plants promoted by Integrase protein of coliphage HK022. Plant Mol Biol 2005;57: 435-444

-Harel-Levi G, Goltsman J, Tuby CN, Yagil E, Kolot M: Human genomic site-specific recom- bination catalyzed by coliphage HK022 integrase. J Biotechnol 2008;134:45-54.

Kolot M, Meroz A, Yagil E: Site-specific recombination in human cells catalyzed by the wild-type integrase protein of coliphage HK022. Biotechnol Bioeng 2003;84:56-60.

Lauth M, Spreafico F, Dethleffsen K, Meyer M: Stable and efficient cassette exchange under non-selectable conditions by combined use of two site-specific recombinases. Nucleic Acids Res 2002;30:e115.

Malchin N, Goltsman J, Dabool L, Gorovits R, Bao Q, Dröge P, Yagil E, Kolot M: Optimization of coliphage HK022 Integrase activity in human cells. Gene 2009;437:9-13.

- Malchin N, Molotsky T, Yagil E, Kotlyar AB, Kolot M: Molecular analysis of recombinasemediated cassette exchange reactions catalyzed by integrase of coliphage HK022. Res Microbiol 2008; 159:663-670.

Qiao J, Oumard A, Wegloehner W, Bode J: Novel tag-and-exchange (RMCE) strategies generate master cell clones with predictable and stable transgene expression properties. J Mol Biol 2009;390:579-594.

Sambrook J, Fritsch EF, Maniatis T: Molecular Cloning: A Laboratory Manual. Cold Spring Harbor, Cold Spring Harbor Laboratory, 1989.

Sorrell DA, Kolb AF: Targeted modification of mammalian genomes. Biotechnol Adv 2005 23:431-469.
- Summers DK, Sherratt DJ: Resolution of ColE1 dimers requires a DNA sequence implicated in the 3-dimensional organization of the cer site. EMBO J 1988;7:851-858.

-Thompson JF, Moitoso de Vargas L, Koch C, Kahmann R, Landy A: Cellular factors couple recombination with growth phase: characterization of a new component in the $\lambda$ site-specific recombination pathway. Cell 1987;50:901-908.

-Vieira J, Messing J: New pUC-derived cloning vectors with different selectable markers and DNA replication origins. Gene 1991;100: 189-194.

-Wallace H, Marques-Kranc ACF, Richardson M, Luna-Crespo F, Sharpe JA, Hughes J, Wood WG, Higgs DR, Smith AJH: Manipulating the mouse genome to engineer precise functional synthetic replacements with human sequence. Cell 2007;128:197-209.

-Weisberg RA, Gottesmann ME, Hendrix RW, Little JW: Family values in the age of genomics: comparative analyses of temperate bacteriophage HK022. Annu Rev Genet 1999;33: 565-602.

Wirth D, Gama-Norton L, Riemer P, Sandhu U, Schucht R, Hauser H: Road to precision: recombinase-based targeting technologies for genome engineering. Curr Opin Biotechnol 2007;18:411-419. 